\title{
Olefin-Polymerisation durch Komplexe später Übergangsmetalle - ein Wegbereiter der Ziegler-Katalysatoren erscheint in neuem Gewand
}

\author{
Stefan Mecking*
}

\section{Einleitung}

Die enorme wirtschaftliche Bedeutung von Polyolefinen spiegelt sich in einer jährlichen Produktion von über 70 Millionen Tonnen Polyethylen und Polypropylen wider. Der größere Teil dieser Materialien wird mittels Ziegler- oder Chrom-Katalysatoren hergestellt, doch hat auch die ältere radikalische Polymerisation zur Synthese von Polyethylen niederer Dichte (LDPE) nach wie vor Bedeutung. ${ }^{[1]}$ Trotz des Erfordernisses von Drücken über 1500 bar werden jährlich 16 Millionen Tonnen LDPE hergestellt, und große Neuanlagen werden weiterhin gebaut. ${ }^{[1 \mathrm{~d}, \mathrm{e}]}$ Ein Vorteil des HochdruckVerfahrens ist die Möglichkeit des Einbaus von funktionalisierten Olefinen wie Acrylaten oder Vinylacetat. Bereits der Einbau eines kleinen Anteils polarer Gruppen kann z.B. die Hafteigenschaften und die Kompatibilität von Polyolefinen mit anderen Materialien deutlich verbessern. Bei der radika-

[*] Dr. S. Mecking

Institut für Makromolekulare Chemie und Freiburger Materialforschungszentrum der Albert-Ludwigs-Universität Freiburg

Stefan-Meier-Straße 31, 79104 Freiburg (Deutschland)

Fax: $(+49) 761-203-6319$

E-mail: mecking@uni-freiburg.de lischen Polymerisation von Ethylen werden, ohne Zusatz eines Comonomers, sowohl Kurz- als auch Langkettenverzweigungen erhalten. Kurzkettenverzweigungen beeinflussen Polymereigenschaften wie die Kristallinität und den Schmelzpunkt und sind ein Schlüssel zur Kontrolle der Anwendungseigenschaften von Polyolefinen. Langkettenverzweigungen (welche typischerweise 100 oder mehr Kohlenstoffatome enthalten) beeinflussen insbesondere die rheologischen Eigenschaften von Polyolefin-Schmelzen und sind der Grund für die gute Verarbeitbarkeit von LDPE.

Diese Beispiele zeigen, dass für die Übergangsmetallkatalysierte Koordinations-Polymerisation im Niederdruckverfahren weiterhin grundlegende Herausforderungen bestehen. Ungünstigerweise sind die auf frühen Übergangsmetallen basierenden Ziegler-Katalysatoren oder Metallocene hochempfindlich gegenüber polaren Reagentien, was sich hinsichtlich des angestrebten Einbaus von polaren Comonomeren als problematisch erweist. Komplexe später Übergangsmetalle weisen dagegen aufgrund ihrer geringeren Oxophilie eine sehr viel höhere Toleranz gegenüber polaren Gruppen auf. Zudem ermöglichen sie den Zugang zu Polyolefinen mit einzigartigen Verzweigungsstrukturen. Verschiedene neue Polymerisations-Katalysatoren auf Basis später 
Übergangsmetalle stellen einen bedeutenden Fortschritt dar. Dieses Highlight gibt, anhand der Polymerisation von Ethylen, einen Überblick über diese Entwicklungen. ${ }^{[2,3]}$

\section{Allgemeiner Hintergrund des Katalysator-Designs}

Die Polymerisation von Ethylen oder $\alpha$-Olefinen mittels Ziegler-Natta- oder Metallocen-Katalyse sowie die C-CVerknüpfung durch Komplexe später Übergangsmetalle verlaufen prinzipiell über die gleichen Typen von Reaktionen, d.h. das Kettenwachstum durch migratorische Insertion in Alkyl-Olefin-Komplexe und den Kettentransfer, für den verschiedene Mechanismen bekannt sind. Aufgrund der Neigung von Alkylkomplexen später Übergangsmetalle zur $\beta$-H-Eliminierung werden mit diesen Komplexen jedoch meist Dimere oder Oligomere als Produkte erhalten (Schema 1). ${ }^{[4,5 c]}$ Ein bekanntes Beispiel ist der Shell-Higher-Olefin-

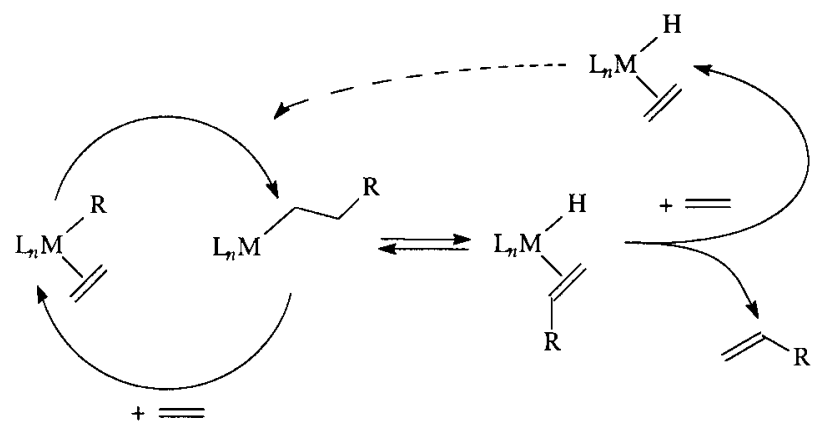

Kettenwachstum

Kettenübertragung durch $\beta$-Hydrideliminierung

Schema 1. Vereinfachtes Schema der Oligomerisierung und Polymerisation von Ethylen durch Komplexe später Übergangsmetalle (M). (R= wachsende Polymer/Oligomer-Kette).

Process(SHOP), welcher durch Keim et al. in den 60er und 70er Jahren entwickelt wurde. Lineare $\alpha$-Olefine werden hier durch die Nickel-katalysierte Oligomerisierung von Ethylen erhalten [Gl. (1)]..[4] Eine Nickel-katalysierte Oligomerisierung von Ethylen kann auch als ein wegbereiter der heutigen Polyolefin-Industrie betrachtet werden: Schließlich führte die Beobachtung, dass die Kombination von Nickel-Spuren mit Aluminiumalkylen die Dimerisierung von Ethylen katalysiert
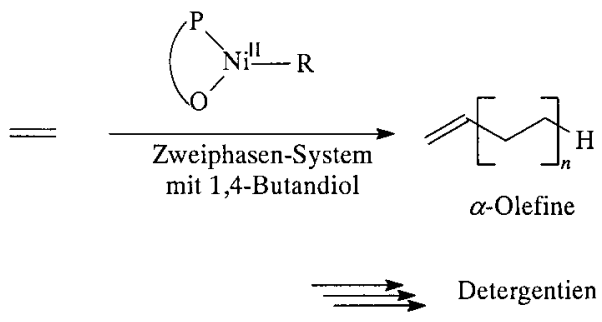

(der berühmte „Nickel-Effekt“), zur Entdeckung der ZieglerKatalysatoren..$^{[5]}$

Ein zentraler Aspekt von Untersuchungen zur Polymerisation durch Komplexe später Übergangsmetalle ist das Design von Katalysatoren, welche die Bildung höhermolekularer Produkte ermöglichen. Im Hinblick auf den Mechanismus bedeutet dies, die Kettenübertragung bei einer gleichzeitig hohen Wachstumsgeschwindigkeit zu unterdrücken (Schema 1). Für eine gegebene Wachstumsgeschwindigkeit bedeutet eine Erhöhung der Barriere für die Kettenübertragung um $\Delta \Delta G^{\ddagger} \approx 3-4 \mathrm{kcal}$ einen Oligomerisierungs-Katalysator in einen Polymerisations-Katalysator umzuwandeln.

Die meisten effektiven Polymerisations-Katalysatoren basieren auf neutralen Nickel(II)-Komplexen mit einem formal monoanionischen zweizähnigen Liganden (Schema 2; 1) oder<smiles>[R][N+]1([3H])CCO1</smiles>

$\widehat{X O}:$ formal monoanionischer zweizähniger Ligand $(\mathrm{X}=\mathrm{P}$ oder $\mathrm{N})$

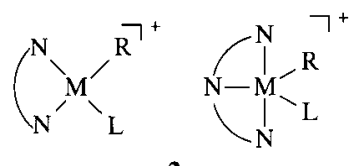

2

$\widehat{\mathrm{NN}}, \widehat{\mathrm{NNN}}$ : neutraler zweibzw. dreizähniger Ligand
Schema 2. Strukturen einiger neutraler und kationischer Komplexe später Übergangsmetalle für die Polymerisation von Ethylen. In wohldefinierten Katalysator-Precursor-Komplexen ist $\mathrm{R}$ ein $\mathrm{Ph}$ - oder Me-Ligand, welcher die Initiierung des Kettenwachstums ermöglicht. L ist ein einzähniger Ligand, meist schwach koordinierend und durch das Monomer verdrängbar. Für die vermuteten aktiven Spezies ist in den meisten Fällen R die wachsende Polymerkette und L das Monomer oder Lösungsmittel.

auf kationischen Eisen-, Cobalt-, Nickel- oder Palladiumkomplexen mit Stickstoff-Donoratomen, die sperrige Substituenten tragen (2). ${ }^{66,7,15,16,21,25,27,28]}$ Das Molekulargewicht der erhaltenen Polymere wird sowohl durch die elektronischen als auch durch die sterischen Eigenschaften am Metallzentrum bestimmt, welche durch den zwei- oder dreizähnigen Liganden gesteuert werden (siehe unten). Die Chelat-Liganden in $\mathbf{1}$ und $\mathbf{2}$ halten das koordinierte Monomer und die wachsende Polymerkette in räumlicher Nähe (z.B in cisStellung in einem quadratisch-planaren Komplex). ${ }^{[7]}$ Zudem enthalten die meisten Katalysatoren keine weiteren stark koordinierenden Liganden (L), welche Koordinationsstellen blockieren und die Bildung niedermolekularer Oligomere begünstigen können. ${ }^{[\mathrm{b}]}$ So wird mit dem typischen SHOPOligomerisierungs-Katalysator $\mathbf{3}^{[4 \mathrm{~b}, \mathrm{c}]}$ in Gegenwart eines Phosphan-Fängers, welcher den Triphenylphosphan-Liganden bindet, lineares Polyethylen erhalten (Schema 3). ${ }^{[16]}$
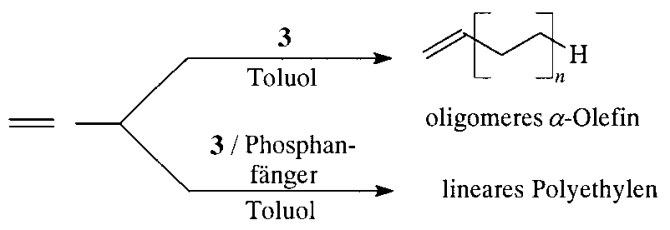<smiles>C1=C(c2ccccc2)O[N+](c2ccccc2)([P+](c2ccccc2)(c2ccccc2)c2ccccc2)C1c1ccccc1</smiles>

3

Schema 3. Effekt eines einzähnigen Triphenylphosphan-Liganden auf das Molekulargewicht der C-C-Verknüpfungsprodukte.

Im Hinblick auf die Umsetzung von Olefinen $\mathrm{zu}$ funktionalisierten Polymeren sei darauf hingewiesen, dass für den speziellen Fall der alternierenden Copolymerisation von Olefinen mit Kohlenmonoxid (Schema 4) durch kationische Palladium(II)-Komplexe eine weitaus größere Bandbreite 

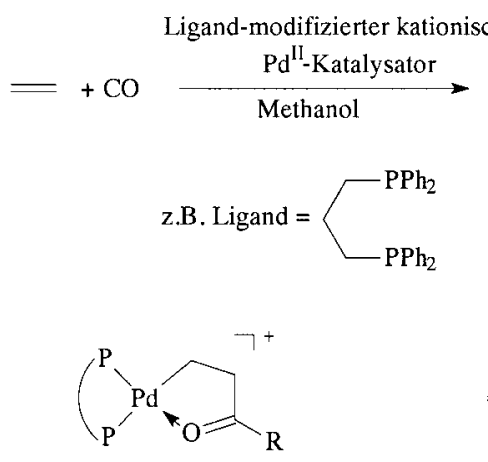

vorgeschlagene Chelatisierung nach einer Ethylen-Insertion

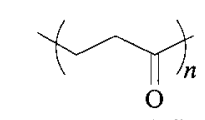

alternierendes Olefin-KohlenmonoxidCopolymer

Schema 4. Copolymerisation von Olefinen mit Kohlenmonoxid und dessen Einfluss auf die Kettenübertragung.

zweizähniger Liganden eingesetzt werden kann. ${ }^{[8]}$ Als Ursache für die Unterdrückung von Kettenübertragungen ist die chelatisierende Koordination der $\beta$-Carbonylgruppe nach einer Ethyleninsertion vorgeschlagen worden, welche eine sterisch ungünstige Anordnung für die $\beta$-H-Eliminierung bewirken kann. ${ }^{[8]}$ Zudem folgt auf eine Ethyleninsertion eine schnelle Insertion von Kohlenmonoxid, sodass ein Acylkomplex (welcher keine $\beta$-H-Eliminierung eingeht) der „Ruhezustand“ des Katalysators ist. ${ }^{[9 c]}$ Dementsprechend katalysieren die Bisphosphan-Komplexe, welche typischerweise für die Ethylen/Kohlenmonoxid-Copolymerisation eingesetzt werden, in Abwesenheit von Kohlenmonoxid die Dimerisierung von Ethylen. ${ }^{[9]}$

Die durch Ethylen/Kohlenmonoxid/Propylen-Terpolymerisation erhaltenen streng alternierenden Polyketone sind chemisch sehr beständig und haben gute Abriebeigenschaften. Die große Zahl an Carbonylgruppen macht diese Polymere jedoch empfindlich gegenüber UV-Strahlung, zudem können bei der Verarbeitung Kondensationsreaktionen zu unerwünschter Quervernetzung führen. Derartige teilkristalline thermoplastische Kunststoffe sind durch Shell seit 1995 produziert worden, die Einstellung dieses Geschäftes wurde jedoch kürzlich angekündigt. ${ }^{[10]}$ Die vinylische Polymerisation von Norbornen kann ebenfalls mit einer Vielzahl von Palladium(II)- und Nickel(II)-Katalysatoren erfolgen. ${ }^{[11]}$ Wiederum tragen sterische Einschränkungen vermutlich wesentlich zur Unterdrückung der Kettenübertragung bei.

\section{Die Vorgeschichte: Polymerisation durch neutrale Nickel(II)-Komplexe mit einem chelatisierenden Liganden}

Beim SHOP wird Ethylen durch neutrale Nickel(II)-Komplexe mit einem chelatisierenden PO-Liganden oligomerisiert [Gl. (1)]. Die Toleranz des Nickel(II)-Katalysators gegenüber polaren Medien ermöglicht die Verwendung von 1,4Butandiol als polare Katalysatorphase. Damit kann industriell ein Zweiphasen-Verfahren angewendet werden, welches eine einfache Abtrennung der apolaren Produkte vom Katalysator ermöglicht. ${ }^{[4 d]}$ Bei der Entwicklung des SHOP sowie in anschließenden grundlegenden Untersuchungen wurde eine Reihe von Katalysatoren für die Polymerisation von Ethylen entdeckt. ${ }^{[4 c, 12,13 b]}$ In der Regel wurde mit moderaten Aktivitäten lineares Polyethylen erhalten (einige $1000 \mathrm{TOh}^{-1} ; M_{\mathrm{v}}$

bis $\mathrm{zu} 10^{5} \mathrm{~g} \mathrm{~mol}^{-1}$, TO $=$ Turnover $=$ mol umgesetztes Substrat je mol Metall). Im Unterschied dazu setzt das Katalysatorsystem $\left[\mathrm{Ni}(\mathrm{cod})_{2}\right] /\left(\mathrm{Me}_{3} \mathrm{Si}\right)_{2} \mathrm{~N}-\mathrm{P}\left(=\mathrm{NSiMe}_{3}\right)_{2}(\operatorname{cod}=1,5-$ Cyclooctadienyl) Ethylen zu einem verzweigten Polymer um. ${ }^{[13]}$ Das Polymer, welches ein relativ niedriges Molekulargewicht aufweist $\left(M_{\mathrm{n}}\right.$ ca. $\left.7 \times 10^{3} \mathrm{~g} \mathrm{~mol}^{-1}\right)$, enthält Methylgruppen sowie längerkettige Verzweigungen. Zur Entstehung dieser überraschenden Mikrostruktur wurde eine gleichzeitige Oligomerisierung und Polymerisation von Ethylen vorgeschlagen, unter Einbau der gebildeten Oligomere in die Polymerkette. ${ }^{[13 \mathrm{~b}, \mathrm{c}]}$ Detaillierte Untersuchungen von Fink et al. zur Polymerisation von $\alpha$-Olefinen durch derartige Katalysatoren zeigten, dass hier eine 2, $\omega$-Polymerisation zu methylverzweigten Polymeren erfolgt. ${ }^{[14]}$ Diese bemerkenswerte Polymerstruktur entsteht durch 1,2-Insertion (Kettenwachstum), gefolgt von einer Wanderung des Nickelzentrums vor der nächsten Insertion [Gl. (2)].

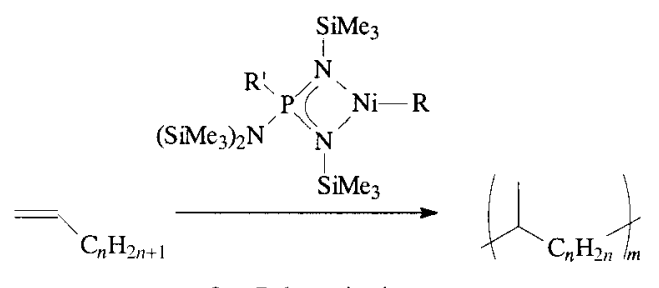

2, $\omega$-Polymerisation

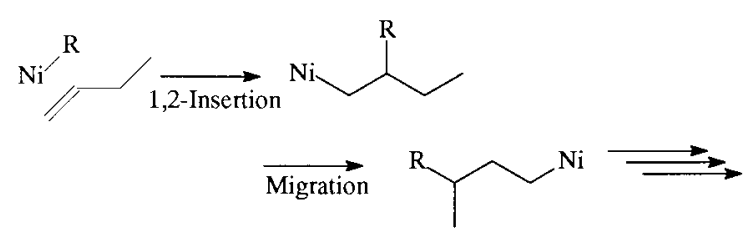

In den 80er Jahren untersuchten Ostoja Starzewski et al. ${ }^{[15]}$ und Klabunde et al. ${ }^{[16]}$ intensiv die Polymerisation von Ethylen durch neutrale Nickel(II)-Komplexe mit P,O-Liganden. Anders als bei 3 (Schema 3) entfällt beim Ersatz des stark koordinierenden Triphenylphosphan-Liganden durch ein schwach koordinierendes Phosphanoxid ${ }^{[15]}$ oder Pyridin ${ }^{[16]}$ die Notwendigkeit eines Phosphan-Fängers. Komplexe mit elektronenreichen Ylid-Liganden $\left(\mathrm{L}=\mathrm{CR}_{2}^{\prime} \mathrm{PR}_{3}\right)$ sind ebenfalls intensiv untersucht worden. ${ }^{[17 a]}$ Diese Nickel(II)-Komplexe stellen aktivatorfreie Einkomponenten-KatalysatorPrecursor für die Ethylenpolymerisation dar. Durch Variation der Ligandenstruktur (z. B. 4 oder 5) sind lineare Polyethylene

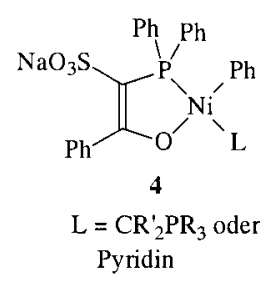<smiles>[Y][N+]1(c2ccccc2)Oc2ccc(O)cc2[PH]1(c1ccccc1)c1ccccc1</smiles>

von sehr hohem Molekulargewicht zugänglich (Tabelle 1). ${ }^{[15,17 b]}$ Typische Polymerisations-Aktivitäten liegen bei $5 \times 10^{4} \mathrm{TOh}^{-1}$, und mit weiter optimierten P,O-Liganden wurden bis $\mathrm{zu} 1.8 \times 10^{5} \mathrm{TOh}^{-1}$ (bei 17 bar Ethylendruck) beobachtet. ${ }^{[18]}$ Diese Katalysatoren sind auch in polaren 
Tabelle 1. Beispiele für die Polymerisation von Ethylen durch Komplexe später Übergangsmetalle.[a]

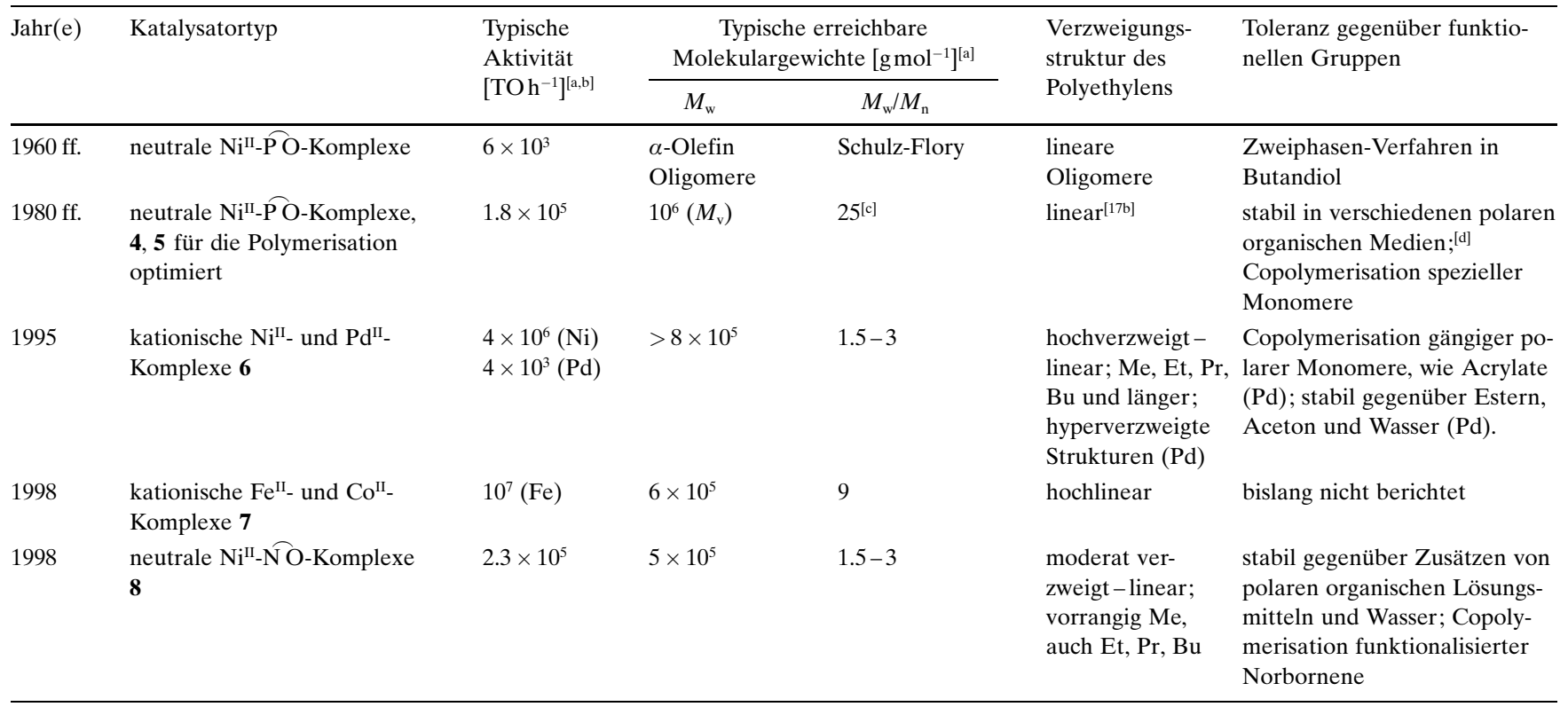

[a] Die Aktivitäten der verschiedenen Katalysatoren wurden unter teilweise stark unterschiedlichen Bedingungen ermittelt, sie sollten daher als Maß für die Größenordnung betrachtet werden. Das gleiche gilt für die Molekulargewichte von Polymeren, welche nach unterschiedlichen Methoden bestimmt wurden. [b] In der Homopolymerisation von Ethylen. [c] Für niedermolekulare Materialien wurden auch enge Verteilungen von $M_{\mathrm{w}} / M_{\mathrm{n}}=2$ berichtet. ${ }^{[15 \mathrm{e}]}[\mathrm{d}]$ Über die Polymerisation unter Verwendung von Wasser als Reaktionsmedium wurde kürzlich berichtet. ${ }^{\text {[29ab] }}$

organischen Reaktionsmedien wie Aceton, DMF oder auch Alkoholen aktiv. $^{[15 b, d, e, ~ 16 b]}$ Versuche zur Copolymerisation von Ethylen mit Methylacrylat oder Vinylacetat waren erfolglos. ${ }^{[16 a]}$ Dagegen ist der Einsatz von Comonomeren wie $\mathrm{H}_{2} \mathrm{C}=\mathrm{CH}-\left(\mathrm{CH}_{2}\right)_{8}-\mathrm{C}(\mathrm{O}) \mathrm{OMe}$, in welchen die polare Gruppe von der Vinylfunktion durch einen „Spacer“ getrennt ist, möglich. ${ }^{[16]}$ Kürzlich wurde auch beschrieben, dass die Copolymerisation von Ethylen mit polar funktionalisierten Norbornenen mit derartigen Nickel(II)Katalysatoren gelingt. ${ }^{[19]}$ Im Hinblick auf die Herstellung verzweigter Polyolefine ist die Oligomerisierung von Ethylen durch Nickel(II)-Katalysatoren mit POChelatliganden und die anschließende Copolymerisation der linearen Oligomere mit Ethylen zu langkettig-verzweigten Polyethylenen beschrieben worden. ${ }^{[15 c, 20]}$

\section{Aktuelle Entwicklungen: kationische Katalysatoren mit mehrzähnigen Liganden}

1995 fand ein Bericht von Brookhart et al. über die Entdeckung einer neuen Klasse von Katalysatoren für die Polymerisation von Ethylen und $\alpha$-Olefinen großes Interesse in industriellen wie auch akademischen Kreisen. ${ }^{[21]}$ Diese Katalysatoren beruhen auf bereits bekannten Palladium(II)- oder Nickel(II)-Komplexen mit Diiminliganden, die sperrige Substituenten haben. ${ }^{[22]}$ Die kationischen Komplexe polymerisieren mit sehr hohen Aktivitäten Ethylen zu hochverzweigten, hochmolekularen Homopolymeren (Schema 5). Für die Nickel(II)-Katalysatoren wurden Aktivitäten von bis $\mathrm{zu}$
$4 \times 10^{6} \mathrm{TOh}^{-1}$ berichtet, welche an die Aktivitäten von Metallocenen heranreichen. ${ }^{[23]}$ Die Molekulargewichtsverteilungen sind relativ eng und liegen im Bereich von Single-SiteKatalysatoren. Wichtig für die Polymerisation zu sehr hohen Molekulargewichten (Tabelle 1) ist die Unterdrückung der Kettenübertragung durch den sterischen Anspruch der $o$ Arylsubstituenten ( $\mathrm{R}=i \operatorname{Pr}$ oder $\mathrm{Me})$. Die Reste $\mathrm{R}$ befinden sich wegen der senkrechten Anordnung der Arylringe oberhalb und unterhalb der (quadratisch-planaren) Koordinationsebene. Zuerst nahm man an, dass die Zurückdrängung der Kettenübertragung an der Behinderung des assoziativen Austausches der ungesättigten Polymerkette vom Metallzentrum durch Ethylen liegt (Schema 6a). Kürzlich durchgeführte Berechnungen zeigen jedoch, dass in den Nickel(II)-Komplexen eine direkte $\beta$-H-Übertragung auf koordiniertes Monomer erfolgen kann, deren Energiebarriere durch sper- 


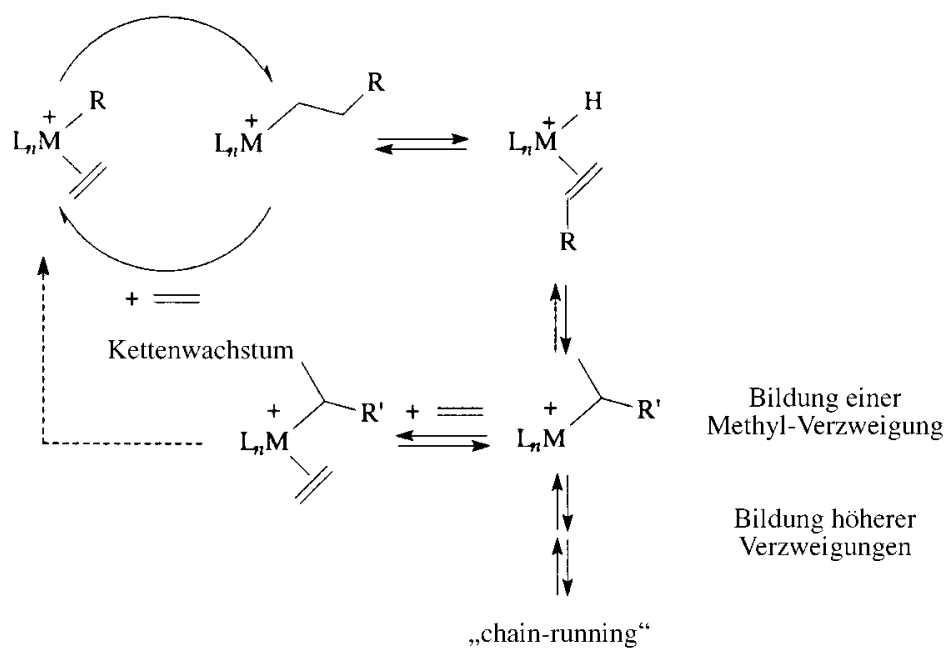

a) Kettenuibertragung durch assoziativen Austausch:

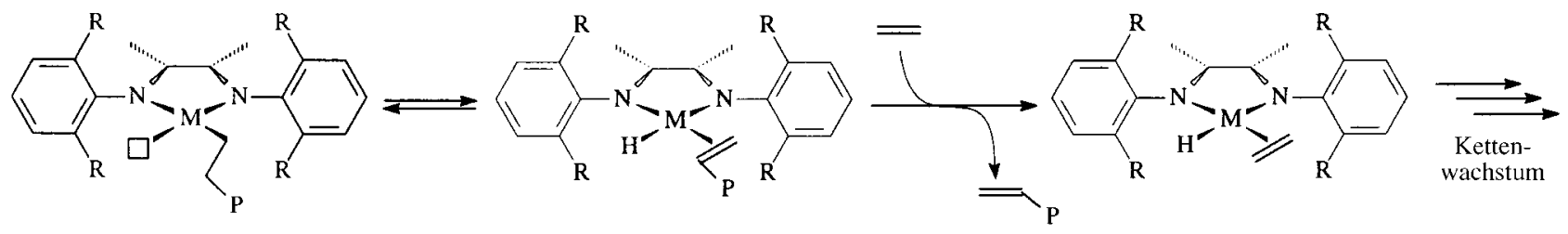

assoziativer Austausch

b) Kettenübertragung zum Monomer:

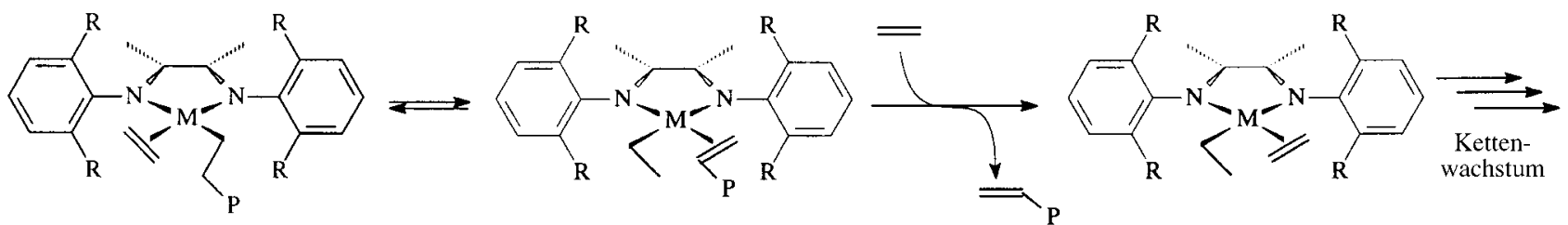

Schema 6. Entstehung von Verzweigungen bei der Polymerisation von Ethylen durch kationische Diimin-substituierte Palladium(II)- und Nickel(II)Katalysatoren. Typische Verzweigungsmuster ${ }^{[21 \mathrm{~h}]}$ (je $1000 \mathrm{CH}_{2}$-Gruppen) mit einem a) Palladium(II)-Katalysator: Me 36, Et 26, Pr 3, Bu 12, Amyl 2, Hexyl und länger sowie Kettenenden 34; b) Nickel(II)-Katalysator: Me 41, Et 6, Pr 3, Bu 2, Amyl 1, Hexyl und länger sowie Kettenenden 5.

rige ortho-Substituenten erhöht wird. ${ }^{[21 g]}$ Die Einführung von weniger sperrigen Substituenten $(\mathrm{R}=\mathrm{H})$ in den Nickel(II)Komplexen führt $\mathrm{zu}$ hochaktiven Katalysatoren für die Oligomerisierung von Ethylen zu $\alpha$-Olefinen. ${ }^{[21 d]}$

Die Polyethylene weisen Verzweigungen mit einer geraden wie auch ungeraden Zahl von Kohlenstoffatomen auf (Me, Et, $\mathrm{Pr}, \mathrm{Bu}$, Amyl, Hexyl und längere Verzweigungen). Mit Nickel(II)-Katalysatoren erhaltene Polymere haben vorwiegend Methylgruppen. Dagegen werden mit den $\mathrm{Pd}^{\mathrm{II}}$-Analoga auch längere Verzweigungen und sogar eine Hyperverzweigung, also eine Verzweigung an einer Verzweigung, gefunden. ${ }^{[21 \mathrm{~h}, \mathrm{j}]}$ Diese Strukturen entstehen durch so genanntes „chainrunning “, das der bereits erwähnten durch Fink untersuchten 2, $\omega$-Polymerisation ähnlich ist (Schema 6). ${ }^{[21 a]}$ Mit den Palladium(II)-Katalysatoren werden unabhängig von der Ethylenkonzentration hochverzweigte amorphe Polymere erhalten (ca. 100 Verzweigungen pro $1000 \mathrm{C}$-Atome), ${ }^{[24]}$ bei den Nickel(II)-Komplexen kann der Verzweigungsgrad dagegen über die Ethylenkonzentration und die Polymerisationstemperatur gesteuert werden (1 bis 100 Verzweigungen pro 1000 C-Ato$\mathrm{me})$. Dementsprechend reichen die makroskopischen Eigenschaften der - aus Ethylen ohne Zusatz von Comonomeren - erhaltenen Polymere von hochviskosen (hochmolekularen) Flüssigkeiten mit $T_{\mathrm{g}}$ von $-70^{\circ} \mathrm{C}$ über gummiartige Elastomere bis hin zu steifen linearen Polyethylenen.

Bemerkenswerterweise ermöglichen die Palladium(II)Komplexe auch die Koordinations-Polymerisation von Ethylen mit Olefinen, die mit polaren Substituenten funktionalisiert sind, z. B. Acrylaten. ${ }^{[21 b, e]}$ Mit zunehmendem Einbau von Acrylat in das Copolymer sinkt die Katalysatoraktivität aufgrund der reversiblen Blockierung des Metallzentrums durch chelatisierende Koordination von Carbonylgruppen des Polymers (Schema 7, Gleichgewicht B). Das „chain-running““ bewirkt auch einen bevorzugten Einbau von Acrylat an den Kettenenden in den hochverzweigten, amorphen Copolymeren. Bislang existiert kein Katalysatorsystem, welches den Einbau polarer Monomere wie Acrylate in die Hauptkette eines linearen Polyethylens ermöglicht.

Die Entdeckung von Brookhart et al. initiierte eine intensive Suche nach anderen geeigneten Ligandenstrukturen. In der Folgezeit wurde von drei Arbeitsgruppen (Brookhart, Gibson und Bennett von DuPont) unabhängig voneinander über neue kationische Eisen- und Cobalt-Katalysatoren des Strukturtyps 7 berichtet. ${ }^{[25 a-d]}$ Mit beeindruckenden Aktivi- 


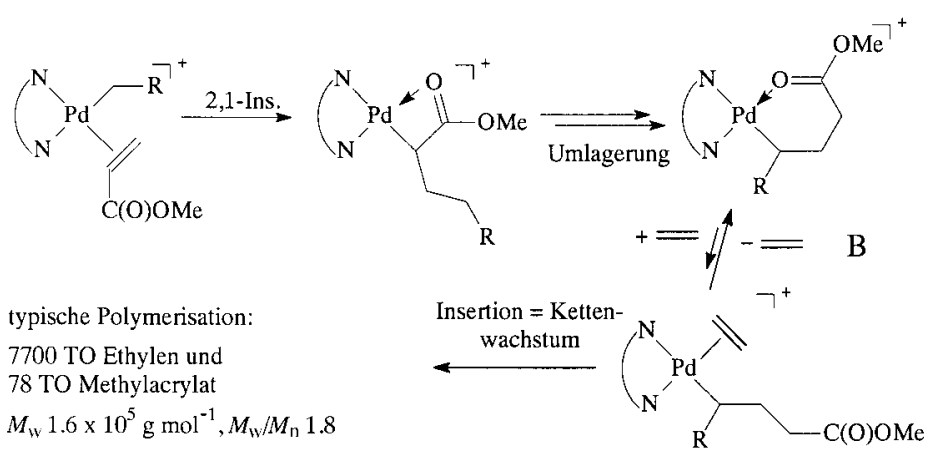

Schema 7. Copolymerisation von Ethylen mit Acrylaten zu hochverzweigten Copolymeren.

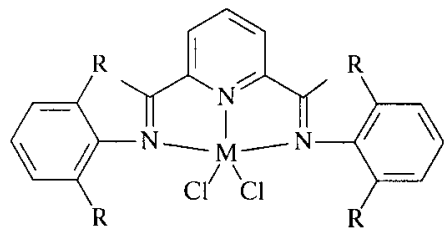

$\mathrm{M}=\mathrm{Fe}, \mathrm{Co}$

$\mathrm{R}=i \mathrm{Pr}, \mathrm{Me}$

Aktivierung z.B. durch MAO

7

täten von bis $\mathrm{zu} 10^{7} \mathrm{TOh}^{-1}$ (40 bar Ethylendruck) wird Ethylen zu hochlinearem, kristallinem HDPE polymerisiert. Die einfachen Synthesen der meisten hier vorgestellten Katalysatoren gehen von kommerziell erhältlichen Verbindungen aus und sind anhand der Eisenkomplexe beispielhaft in Gleichung (3) dargestellt. Da zusätzlich zur $\beta$-H-Eliminierung Kettenübertragung auf den aluminiumhaltigen Cokata-<smiles>[R]c1cccc([R])c1N</smiles><smiles>[R]C(=Nc1c([R])cccc1[R])c1cccc(-c2nc3c([R])cccc3[R]2[H])n1</smiles>

lysator erfolgt, werden mit den Eisen-Katalysatoren Polymere von breiter oder bimodaler Molekulargewichtsverteilung erhalten (Tabelle 1). Bislang sind keine EinkomponentenKatalysator-Precursor als Alternative zu den Aluminiumalkyl-aktivierten In-situ-Systemen bekannt. BP-Amoco und DuPont haben eine Vereinbarung mit dem Ziel der Kommerzialisierung der Eisen-katalysiert hergestellten steifen Polyethylene angekündigt. ${ }^{[25]}$ In Analogie zu den bereits erwähnten Diimin-substituierten Nickel(II)- und Palladium(II)Komplexen unterdrücken die sterisch anspruchsvollen $o$ Arylsubstituenten die Kettenübertragung. Eine Verringerung des sterischen Anspruches der Substituenten R in den EisenKatalysatoren führt wiederum zu Katalysatoren für die Oligomerisierung von Ethylen. ${ }^{[25 e, f]}$ Extrem hohe Aktivitäten von bis zu $10^{8} \mathrm{TOh}^{-1}$ für die Umsetzung $\mathrm{zu}>99 \%$ linearen $\alpha$-Olefinen wurden beobachtet, was die Aktivitäten der bekannten SHOP-Katalysatoren weit übersteigt. Die Suche nach neuen Polymerisations-Katalysatoren und das gewonnene grundlegende Verständnis führt interessanterweise wiederum zu hochaktiven neuen Systemen für die Oligomerisierung.

\section{Neue neutrale Nickel(II)-Katalysatoren mit $\overparen{N O}$-Chelatliganden}

Etwa zeitgleich mit den kationischen Eisen- und CobaltKatalysatoren wurde von Johnson ${ }^{[27]}$ und Grubbs ${ }^{[28]}$ unabhängig voneinander über einen neuen Typ von neutralen Nickel(II)-Katalysatoren $\mathbf{8}$ mit Salicylaldimin-Liganden berichtet. ${ }^{[26]}$ Hinsichtlich des formal monoanionischen zweizähnigen Liganden ähneln diese Komplexe den SHOP-Katalysatoren. Der P-Donor der letzteren ist jedoch durch eine substituierte<smiles>[X]c1cc([R])c2c(c1)c([R])n(-c1c([R])cccc1[R])[n+]2P</smiles>

$8 \mathrm{a}$<smiles>C=CC(=C)C(=O)OC</smiles>

$8 b$
Imin-Funktion mit sperrigem Rest ersetzt. Mit diesen Katalysatoren werden wenig verzweigte bis lineare Polyethylene erhalten. Es werden ähnlich hohe gewichtsmittlere Molmassen erzielt wie mit herkömmlichen auf P,O-Liganden basierenden Katalysatoren erhaltenen Materialien, bei jedoch wesentlich engerer Molekulargewichtsverteilung (Tabelle 1). Anfangs wurden substituierte Allylkomplexe ${ }^{[27]} \mathbf{8 b}$ oder Triphenylphosphankomplexe ${ }^{[28]}$ 8a eingesetzt, welche eine Lewis-Säure oder einen Phosphan-Fänger als Cokatalysator erfordern. Die Einführung sterisch anspruchsvoller Gruppen $\mathrm{R}^{\prime}$ in ortho-Stellung zur Phenolatfunktion ermöglicht die Verwendung der Phosphankomplexe als EinkomponentenPrecursor ohne Zusatz eines Phosphan-Fängers, da die Dissoziation des Triphenylphosphan-Liganden gefördert wird. ${ }^{[28 b]}$ Methylkomplexe mit schwach koordinierenden Acetonitril-Liganden, welche nach Gleichung (4) hergestellt wer-

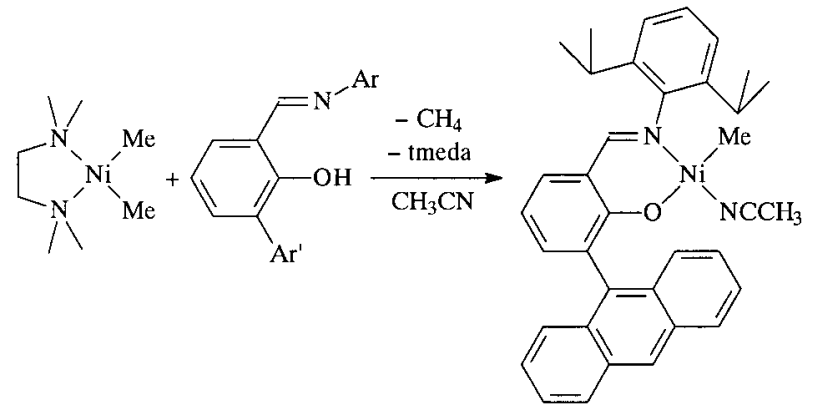

den können, stellen vielseitige Einkomponenten-Precursor dar $\quad\left(\right.$ tmeda $=N, N, N^{\prime} N^{\prime}$-Tetramethylethylendiamin $) .{ }^{[27,28 \mathrm{~b}, \mathrm{c}]}$ Die Aktivitäten in der Ethylen-Homopolymerisation $(2.3 \times$ $10^{5} \mathrm{TO} \mathrm{h}^{-1}$ bei $\left.17 \mathrm{bar}\right)^{[28 \mathrm{~b}]}$ entsprechen denen optimierter Polymerisations-Katalysatoren auf P,O-Liganden-Basis. Wie die P,O-substituierten neutralen Nickel(II)-Katalysatoren sind 
die auf N,O-Liganden basierenden Systeme tolerant gegenüber funktionellen Gruppen. Grubbs et al. haben Ethylen mit 5-funktionalisierten Norbornenen copolymerisiert [Gl. (5)], ${ }^{[28 b, c]}$ und in der Ethylenhomopolymerisation wurden

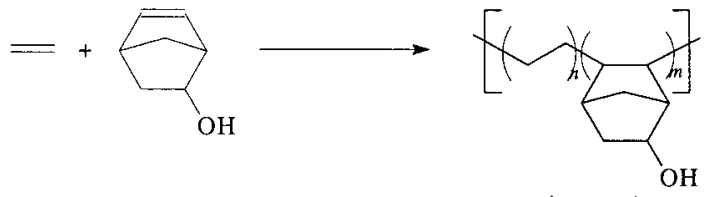

typisches Ergebnis: $M_{\mathrm{W}} 1.7 \times 10^{4} \mathrm{~g} \mathrm{~mol}^{-1}$

460 TO Ethylen, 34 TO funktionalisiertes Norbornen

hochmolekulare Polymere auch in Gegenwart polarer Reagentien wie Ethern, Ethylacetat, Aceton oder auch Wasser erhalten. ${ }^{[28 b]}$ Derartige Polymerisationen in Gegenwart zugesetzter polarer Reagentien sind für die Polymerisation von weniger aufwendig gereinigten Monomeren von Interesse. ${ }^{\text {[28b] }}$

Völlig andere Möglichkeiten bietet die Verwendung von Wasser als Reaktionsmedium. Die einzigartigen Eigenschaften von Wasser sind die Grundlage für die radikalische Emulsions- oder Suspensionspolymerisation olefinischer Monomere. Damit können z. B. Polymerlatices direkt hergestellt werden. Neuere Untersuchungen ergaben, dass derartige Reaktionen auch durch vinylische Koordinations-Polymerisation mit Komplexen später Übergangsmetalle als Katalysatoren möglich sind. ${ }^{[29,6]}$

Zusammenfassend lässt sich sagen, dass in jüngster Zeit bedeutende Fortschritte im Bereich der Olefinpolymerisation durch späte Übergangsmetalle als Katalysatoren erzielt wurden. Polyolefine mit besonderen Mikrostrukturen sind zugänglich geworden, und die Copolymerisation einfacher polarer Comonomere ist möglich. Zudem haben mechanistische Untersuchungen das grundlegende Verständnis dieser Reaktionen stark erweitert. Diese Einblicke haben bereits zum rationellen Design neuer Katalysatoren geführt, z.B. neutrale Nickel(II)-Komplexe mit Salicylaldimin-Liganden. Die verfügbaren Daten zu den Anwendungseigenschaften der neuen Materialien erlauben bislang kein schlüssiges Gesamtbild. Angesichts des großen Interesses an diesen Polymeren wird sich dies in der nahen Zukunft sicherlich ändern.

[1] a) Ziegler Catalysts (Hrsg.: G. Fink, R. Mülhaupt, H. H. Brintzinger), Springer, Berlin, 1995; b) H. H. Brintzinger, D. Fischer, R. Mülhaupt, B. Rieger, R. Waymouth, Angew. Chem. 1995, 107, 1255-1283; Angew. Chem. Int. Ed. Engl. 1995, 34, $1143-1170 ;$ c) W. Kaminsky, M. Arndt, Adv. Polym. Sci. 1997, 127, 143-187; d) M. Balsam, C. Lach, R.-D. Maier, Nachr. Chem. 2000, 48, 338-347; e) K. S. Whiteley in Ullmann's Encyclopedia of Industrial Chemistry, Vol. A21, 5. Aufl. (Hrsg.: W. Gerhartz, B. Elvers), VCH, Weinheim, 1992, S. 488-517.

[2] Übersichtsartikel zur Olefinpolymerisation durch Komplexe später Übergangsmetalle: a) G. J. P. Britovsek, V. C. Gibson, D. F. Wass, Angew. Chem. 1999, 111, 448-468; Angew. Chem. Int. Ed. 1999, 38, $428-447$; b) S. D. Ittel, L. K. Johnson, M. Brookhart, Chem. Rev. 2000, 100, 1169-1204; c) S. Mecking, Coord. Chem. Rev. 2000, 203, 325-351; d) A. S. Abu-Surrah, B. Rieger, Angew. Chem. 1996, 108, 2427-2429; Angew. Chem. Int. Ed. Engl. 1996, 35, 2475-2477.

[3] Die Polymerisation von Dienen, cyclischen Olefinen, Acetylenen und $\alpha$-Olefinen, Copolymerisationen von Olefinen mit Kohlenmonoxid sowie die Ringöffnungs-Metathesepolymerisation werden in diesem Beitrag nicht weiter behandelt.
[4] a) M. Peuckert, W. Keim, Organometallics 1983, 2, 594-597; b) W. Keim, F. H. Kowaldt, Erdöl Erdgas Kohle 1978, 78-79, 453-462; c) W. Keim, F. H. Kowaldt, R. Goddard, C. Krüger, Angew. Chem. 1978, 90, 493; Angew. Chem. Int. Ed. Engl. 1978, 17, 466-467; d) D. Vogt in Aqueous-Phase Organometallic Chemistry (Hrsg.: B. Cornils, W. A. Herrmann), Wiley-VCH, Weinheim, 1998, S. $541-$ 547.

[5] a) K. Ziegler, E. Holzkamp, H. Breil, H. Martin, Angew. Chem. 1955, 67, 541-547; b) K. Fischer, K. Jonas, P. Misbach, R. Stabba, G. Wilke, Angew. Chem. 1973, 85, 1002-1012; Angew. Chem. Int. Ed. Engl. 1973, 12, 943 -953; c) G. Wilke, Angew. Chem. 1988, 100, 189-211; Angew. Chem. Int. Ed. Engl. 1988, 27, 185-206.

[6] Zur langsamen (1 TO pro Tag) Rhodium-katalysierten Polymerisation von Ethylen in Wasser: L. Wang, R. S. Lu, R. Bau, T. C. Flood, J. Am. Chem. Soc. 1993, 115, 6999-7000.

[7] Für die Ethylenpolymerisation sind auch einige Katalysatoren mit sterisch anspruchsvollen einzähnigen Liganden beschrieben worden, ein frühes Beispiel findet sich in Lit. [5c].

[8] Übersichtsartikel: a) E. Drent, P. H. M. Budzelaar, Chem. Rev. 1996 96, 663-681; b) A. Sen, Acc. Chem. Res. 1993, 26, 303-310; c) A. S. Abu-Surrah, B. Rieger, Top. Catal. 1999, 7, 165-177.

[9] a) E. Drent, J. A. M. van Broekhoven, M. J. Doyle, J. Organomet. Chem. 1991, 417, 235-251; b) siehe auch: A. Sen, T. W. Lai, J. Am. Chem. Soc. 1982, 104, 3520-3522; c) F. C. Rix, M. Brookhart, P. S. White, J. Am. Chem. Soc. 1996, 118, 4746-4764.

[10] a) N. Alperowicz, Chem. Week 1995, July 2, 22; b) Anonymous, Chem. Eng. News 2000, 78(9), 16

[11] a) S. Breunig, W. Risse, Makromol. Chem. 1992, 193, 2915-2927; b) B. L. Goodall, D. A. Barnes, G. H. Benedikt, L. H. McIntosch, Proceedings of the 6th International Business Forum on Specialty Polyolefins (SPO'96), Schottland Business Research, 1996, S. 121 142 ; c) A. L. Safir, B. M. Novak, Macromolecules 1995, 28, $5396-$ 5398 ; d) B. S. Heinz, W. Heitz, S. A. Krügel, F. Raubacher, J. H. Wendorff, Acta Polym. 1997, 48, 385-391.

[12] a) R. Bauer, H. Chung, G. Cannell, W. Keim, H. van Zwet (Shell), USPat. 3637636, 1972 [Chem. Abstr. 1971, 75, 130322r]; b) R. Bauer, H. Chung, K. W. Barnett, P. W. Glockner, W. Keim (Shell), US-Pat. 3686159, 1972 [Chem. Abstr. 1972, 76, 15196m].

[13] a) W. Keim, R. Appel, A. Storeck, C. Krüger, R. Goddard, Angew. Chem. 1981, 93, 91-92; Angew. Chem. Int. Ed. Engl. 1981, 20, 116117; b) W. Keim, Ann. N.Y. Acad. Sci. 1983, 415, 191-200; c) A. Storeck, Dissertation, RWTH Aachen, 1980.

[14] a) V. M. Möhring, G. Fink, Angew. Chem. 1985, 97, 982-984; Angew. Chem. Int. Ed. Engl. 1985, 24, 1001-1003; b) R. Schubbe, K. Angermund, G. Fink, R. Goddard, Macromol. Chem. Phys. 1995, 196, 467-478.

[15] a) K. A. Ostoja Starzewski, J. Witte, Angew. Chem. 1985, 97, 610-612; Angew. Chem. Int. Ed. Engl. 1985, 24, 599-601; b) K. A. Ostoja Starzewski, J. Witte, Angew. Chem. 1987, 99, 76-77; Angew. Chem. Int. Ed. Engl. 1987, 26, 63-64; c) K. A. Ostoja Starzewski, J. Witte, K. H. Reichert, G. Vasiliou in Transition Metals and Organometallics as Catalysts for Olefin Polymerization (Hrsg.: W. Kaminsky, H. Sinn), Springer, Berlin, 1988, S. 349-360; d) K. H. A. Ostoja Starzewski, J. Witte, H. Bartl, DE-A 3336500, 1985 [Chem. Abstr. 1985, 103, 142517t]; e) K. A. Ostoja Starzewski, J. Witte in Transition Metal Catalyzed Polymerizations - Ziegler Natta and Metathesis Polymerization (Hrsg.: R. P. Quirk), Cambridge University Press, Cambridge, 1988, S. $472-496$.

[16] a) U. Klabunde, S. D. Ittel, J. Mol. Catal. 1987, 41, 123-134; b) U. Klabunde, R. Mülhaupt, T. Herskovitz, A. H. Janowicz, J. Calabrese, S. D. Ittel, J. Polym. Sci. Part A 1987, 25, 1989-2003; c) U. Klabunde (DuPont), US-Pat. 4698403, 1987 [Chem. Abstr. 1988, 108, $151134 w]$.

[17] a) Das Auftreten von fünffach koordinierten aktiven Spezies, in welchen der Ylid-Ligand an das Metallzentrum koordiniert, ist denkbar. b) Die Angaben zur Polymerstruktur belegen eine lineare Struktur. ${ }^{[15,16]}$ In Oligomeren, welche mit Ylid-substituierten Katalysatoren bei hohen Katalysator-Konzentrationen erhalten wurden, sind auch Vinyliden-Gruppen identifiziert worden. ${ }^{[15 c]}$

[18] z. B. K. Kurtev, A. Tomov, J. Mol. Catal. 1994, 88, 141-150. 
[19] K. L. Makovetsky, E. S. Finkelstein, V. I. Bykov, A. K. Bagdasaryan, B. L. Goodall, L. F. Rhodes (BFGoodrich), WO 98/56837, 1998 [Chem. Abstr. 1999, 130, 66910f].

[20] Zur historischen Entwicklung der simultanen Oligomerisierung/ Copolymerisation von Ethylen siehe Fußnote 175 in Lit. [2b]; siehe auch: D. L. Beach, Y. V. Kissin, J. Polym. Sci. Polym. Chem. 1984, 22, 3027-3042 (Kurzkettenverzweigung).

[21] a) L. K. Johnson, C. M. Killian, M. Brookhart, J. Am. Chem. Soc. 1995, 117, 6414-6415; b) L. K. Johnson, S. Mecking, M. Brookhart, J. Am. Chem. Soc. 1996, 118, 267-268; c) C. M. Killian, D. J. Tempel, L. K. Johnson, M. Brookhart, J. Am. Chem. Soc. 1996, 118, $11664-$ 11665 ; d) C. M. Killian, L. K. Johnson, M. Brookhart, Organometallics 1997, 16, 2005-2007; e) S. Mecking, L. K. Johnson, L. Wang, M. Brookhart, J. Am. Chem. Soc. 1998, 120, 888-899; f) S. A. Svejda, L. K. Johnson, M. Brookhart, J. Am. Chem. Soc. 1999, 121, $10634-$ 10635 ; g) D. P. Gates, S. A. Svejda, E. Onate, C. M. Killian, L. K. Johnson, P. S. White, M. Brookhart, Macromolecules 2000, 33, 2320 2334, zit. Lit.; h) L. K. Johnson, C. M. Killian, S. D. Arthur, J. Feldman, E. McCord, S. J. McLain, K. A. Kreutzer, M. A. Bennett, E. B. Coughlin, S. D. Ittel, A. Parthasarathy, D. Tempel, M. Brookhart (UNC-Chapel Hill/DuPont), WO 96/23010, 1996 [Chem. Abstr. 1996, 125, 222773t]; j) Z. Guan, P. M. Cotts, E. F. McCord, S. J. McLain, Science 1999, 283, 2059-2062.

[22] Derartige Diimin-Komplexe sind intensiv untersucht worden: a) G. van Koten, K. Vrieze, Adv. Organomet. Chem. 1982, 21, 151-239; b) R. van Asselt, E. E. C. G. Gielens, R. Ruelke, K. Vrieze, C. J. Elsevier, J. Am. Chem. Soc. 1994, 116, 977-985; c) H. tom Dieck, M. Svoboda, T. Greiser, Z. Naturforsch. B 1981, 36, 823-832.

[23] Ein aktueller Überblick über die Aktivitäten von Metallocenen in der Ethylenpolymerisation findet sich in H. G. Alt, A. Köppl, Chem. Rev. 2000, 100, 1205-1222 sowie in Lit. [1c].

[24] Auch im Falle der Palladium(II)-Katalysatoren kann die Ethylenkonzentration den Anteil der längeren Verzweigungen, und damit die Polymereigenschaften, beeinflussen. ${ }^{[21 \mathrm{~g}, \mathrm{j}]}$
[25] a) B. L. Small, M. Brookhart, A. M. A. Bennett, J. Am. Chem. Soc 1998, 120, 4049-4050; b) G. J. P. Britovsek, V. Gibson, B. S. Kimberley, P. J. Maddox, S. J. McTavish, G. A. Solan, A. J. P. White, D. J. Williams, Chem. Commun. 1998, 849-850; c) A. M. A. Bennett (DuPont), WO 98/27124, 1998 [Chem. Abstr. 1998, 129, 122973x]; d) G. J. P. Britovsek, M. I. Bruce, V. C. Gibson, B. S. Kimberley, P. J Maddox, S. Mastroianni, S. J. McTavish, C. Redshaw, G. A. Solan, S. Strömberg, A. J. P. White, D. J. Williams, J. Am. Chem. Soc. 1999, 121 8728-8740; e) B. L. Small, M. Brookhart, J. Am. Chem. Soc. 1998, 120, 7143-7144; f) G. J. P. Britovsek, S. Mastroianni, G. A. Solan, S. P. D. Baugh, C. Redshaw, V. C. Gibson, A. J. P. White, D. J Williams, M. R. J. Elsegood, Chem. Eur. J. 2000, 6, 2221-2231; g) Anonymous, Chem. Eng. News 2000, 78(8), 9.

[26] Die Ethylenpolymerisation durch andere N,O-substituierte (PyridinCarboxylato) Nickel(II)-Komplexe, welche mit relativ niedrigen Aktivitäten erfolgt, ist zuvor beschrieben worden: S. Y. Desjardins, K. J. Cavell, J. L. Hoare, B. W. Skelton, A. N. Sobolev, A. H. White, W. Keim, J. Organomet. Chem. 1997, 544, 163-174.

[27] L. K. Johnson, A. M. A. Bennett, S. D. Ittel, L. Wang, A. Parthasarathy, E. Hauptman, R. D. Simpson, J. Feldman, E. B. Coughlin (DuPont), WO 98/30609, 1998 [Chem. Abstr. 1998, 129, 149362j].

[28] a) C. Wang, S. Friedrich, T. R. Younkin, R. T. Li, R. H. Grubbs, D. A. Bansleben, M. W. Day, Organometallics 1998, 17, 3149-3151; b) T. R. Younkin, E. F. Connor, J. I. Henderson, S. K. Friedrich, R. H. Grubbs, D. A. Bansleben, Science 2000, 287, 460-462; c) R. H. Grubbs, Vortrag auf dem 219. ACS National Meeting, 2000 (paper 230).

[29] Ethylenpolymerisation: a) A. Held, F. M. Bauers, S. Mecking, Chem. Commun. 2000, 301-302; b) A. Tomov, J.-P. Broyer, R. Spitz Macromol. Symp. 2000, 150, 53-58. Copolymerisation von Ethylen mit Kohlenmonoxid: c) Z. Jiang, A. Sen, Macromolecules 1994, 27, 7215 -7216; d) G. Verspui, G. Papadogianakis, R. A. Sheldon, Chem. Commun. 1998, 401-402; e) C. Bianchini, H. Man Lee, A. Meli, S. Moneti, V. Patinec, G. Petrucci, F. Vizza, Macromolecules 1999, 32 3859 -3866; f) G. Verspui, F. Schanssema, R. A. Sheldon, Angew. Chem. 2000, 112, 825-827; Angew. Chem. Int. Ed. 2000, 39, 804-806. 